ARTICLE

\title{
Generation of a Gluconobacter oxydans knockout collection for improved extraction of rare earth elements
}

\author{
Alexa M. Schmitz', Brooke Pian (1) ${ }^{1}$, Sean Medin', Matthew C. Reid², Mingming Wu (D) ${ }^{1}$, Esteban Gazel (D) ${ }^{3}$ \& \\ Buz Barstow (1) ${ }^{1 \times}$
}

Bioleaching of rare earth elements (REEs), using microorganisms such as Gluconobacter oxydans, offers a sustainable alternative to environmentally harmful thermochemical extraction, but is currently not very efficient. Here, we generate a whole-genome knockout collection of single-gene transposon disruption mutants for $\mathrm{G}$. oxydans B58, to identify genes affecting the efficacy of REE bioleaching. We find 304 genes whose disruption alters the production of acidic biolixiviant. Disruption of genes underlying synthesis of the cofactor pyrroloquinoline quinone (PQQ) and the PQQ-dependent membrane-bound glucose dehydrogenase nearly eliminates bioleaching. Disruption of phosphate-specific transport system genes enhances bioleaching by up to $18 \%$. Our results provide a comprehensive roadmap for engineering the genome of $G$. oxydans to further increase its bioleaching efficiency.

\footnotetext{
${ }^{1}$ Department of Biological and Environmental Engineering, Cornell University, Ithaca, NY 14853, USA. ${ }^{2}$ School of Civil and Environmental Engineering, Cornell University, Ithaca, NY 14853, USA. ${ }^{3}$ Department of Earth and Atmospheric Sciences, Cornell University, Ithaca, NY 14853, USA.

凶email: bmb35@cornell.edu
} 
$\mathrm{R}$ are earth elements (REEs) are essential for the manufacturing of modern electronics ${ }^{1-3}$ and sustainable energy technologies, including electric motors and wind turbine generators ${ }^{4}$, solidstate lighting 5 , battery anodes ${ }^{6}$, high-temperature superconductors ${ }^{7}$, and high-strength lightweight alloys ${ }^{8,9}$. All of these applications place increasing demands on the global REE supply chain ${ }^{10}$. As the world demand for sustainable energy grows ${ }^{11}$, finding a reliable and sustainable source of REEs is critical.

Current methods for refining REEs often involve harsh chemicals, high temperatures, and high pressures and generate a considerable amount of toxic waste ${ }^{12}$. These processes give sustainable energy technologies reliant on REEs a high environmental and carbon footprint. As a consequence, due to its high environmental standards, the United States has no capacity to produce purified REEs ${ }^{13,14}$.

There is growing interest in biological methods to supplement, if not completely replace, traditional REE extraction and purification methods ${ }^{15-19}$. Biological extraction (bioleaching) is already used to extract $5 \%$ of the world's gold 20,21 and $\approx 15 \%$ of the world's copper supply 20,21 (in fact, Cu biomining in Chile alone accounts for $10 \%$ of the world's $\mathrm{Cu}$ supply ${ }^{22,23}$ ).

The performance of REE bioleaching lags behind thermochemical processes. For example, while thermochemical methods have $89-98 \%$ REE extraction efficiency from monazite ore ${ }^{24,25}$, Aspergillus species can only achieve $\approx 3-5 \%{ }^{16}$. The acid-producing microbe Gluconobacter oxydans $\mathrm{B} 58$ can recover $\approx 50 \%$ of REEs from FCC catalysts ${ }^{26}$. However, techno-economic analysis indicates that even this extraction efficiency is still not high enough for commercial application ${ }^{19}$.

Recent efforts to improve bioleaching have focused exclusively on process optimization ${ }^{27}$. To our knowledge, no genetic approaches have yet been taken for any bioleaching microbe ${ }^{28}$. With recent advances in tools for reading and writing genomes, genetic engineering is an attractive solution for enhancing bioleaching. However, applying these tools to non-model microorganisms such as $G$. oxydans can be a significant challenge ${ }^{9}$. While there have been some promising advances in editing the $G$. oxydans genome ${ }^{29-34}$, we do not yet know where to edit.

In the presence of glucose, $G$. oxydans secretes a biolixiviant rich in gluconic acid ${ }^{26}$. This is produced by periplasmic glucose oxidation by the pyrroloquinoline quinone (PQQ)-dependent membrane-bound glucose dehydrogenase $(\mathrm{mGDH})^{35}$. The final $\mathrm{pH}$ of the biolixiviant is a major factor in REE bioleaching ${ }^{26}$. However, gluconic acid alone fails to explain bioleaching by $G$. oxydans: pure gluconic acid is far less effective at bioleaching than the biolixiviant produced by G. oxydans ${ }^{26}$. This means that even the most successful efforts to upregulate mGDH activity and gluconic acid production are unlikely to take full advantage of $G$. oxydans' biolixiviant production capabilities.

In this work, we characterize the genome of G. oxydans and identify a comprehensive set of genes underlying its bioleaching capabilities through the construction of a carefully curated wholegenome knockout collection of single-gene transposon disruption mutants using Knockout Sudoku ${ }^{36,37}$ (Fig. 1). As the final pH of the biolixiviant is a good predictor of bioleaching efficiency ${ }^{26}$; we use acidification as a proxy for bioleaching potential and screen the collection for mutants that differ in their ability to produce acidic biolixiviant (Figs. 2 and 3). Finally, we demonstrate that a single gene disruption - only one of several potential enhancement strategies-can already significantly improve G. oxydans' bioleaching capabilities (Fig. 4).

\section{Results}

Development of a knockout collection for G. oxydans. We built a saturating coverage transposon insertion mutant collection for $G$. oxydans B58 and cataloged and condensed it with the Knockout Sudoku combinatorial pooling method ${ }^{36,37}$ (Fig. 1). We sequenced the G. oxydans B58 genome and identified 3283 open reading frames (Fig. 1A and Supplementary Data 1; see "Methods" section). Following the recommendation of Monte Carlo simulations, we collected 49,256 transposon insertion mutants (the progenitor collection; PC) to ensure saturating coverage of the $G$. oxydans B58 genome (Fig. 1B; see "Methods" section).

Progenitor collection sequencing results indicate that we were able to generate at least one disruption mutant for almost every nonessential gene in the $G$. oxydans genome. In total, we identified disruption strains for 2733 genes out of the 3283 genes in the $G$. oxydans B58 genome. Since every predicted gene contains at least seven AT or TA transposon insertion sites (Supplementary Data 1), the remaining 550 non-disrupted genes are likely to be essential. A Fisher's Exact Test for gene ontology (GO) enrichment representing 268 of the non-disrupted genes demonstrated significant enrichment $(p<0.05)$ in several essential ontologies, with the greatest enrichment in those relating to the ribosome and translation (Fig. $1 \mathrm{C}$ and Supplementary Data 2A).

The progenitor collection catalog was used to create a condensed G. oxydans disruption collection with at least one representative per nonessential gene. Forty-seven progenitor strains were verified by Sanger sequencing prior to condensing, of which 43 (92\%) were confirmed to have the predicted transposon coordinate (Supplementary Data 3A). We selected one mutant for all 2733 disrupted genes, a second mutant for 2354 genes, and a third mutant for 50 genes where mutant location information was poor. All mutants were transferred for isolation of single colonies, and 2-10 colonies per mutant were picked, depending on the predicted number of cross-contaminating disruption strains in the originating well. This condensed collection contains 17,706 mutants in 185 96-well plates (Supplementary Data 4).

The condensed collection catalog was validated by a second round of combinatorial pooling and sequencing. Of the 17,706 wells in the condensed collection, we were able to confirm the identity of 15,257 (Supplementary Data 4). We confirmed 25 of these wells by Sanger sequencing, and $100 \%$ had the predicted transposon coordinates (Supplementary Data 3B). Among these wells, we were able to verify the identity and location of 4419 independent transposon insertion sites, representing a disruption mutant for 2556 unique genes (Fig. 1D). One thousand five hundred and eighty seven genes are represented by more than one disruption, and 3317 of all disruptions occur in the first half of the gene (Supplementary Data 4).

Genome-wide screening discovers genes linked to acid production. We screened the G. oxydans B58 whole genome knockout collection for disruption mutants with differential acidification capability (Fig. 2). We used the colorimetric pHsensitive dye thymol blue (TB) to screen for changes in final biolixiviant $\mathrm{pH}$ (Fig. 2A and $\mathrm{S} 1$ ) and bromophenol blue (BPB) to screen for changes in the rate of acidification (Fig. 2B).

In total, we noted 304 genes that apparently controlled acidification (Fig. 2C and Supplementary Data 5). The TB screen discovered 282 genes whose disruption led to a differential change in biolixiviant acidity (Fig. 2C and Supplementary Data 5). Fortyseven mutants produced a more acidic biolixiviant, while 235 produced a less acidic biolixiviant (Fig. 2C and Supplementary Data 5). The BPB screen identified 82 gene disruptions with differential rates of acidification: 49 with a faster rate and 33 with a slower rate. Sixty mutants were identified by both screens (Fig. 2C and Supplementary Data 5).

Overall, we identified 165 genes that significantly $(p<0.05)$ changed the final biolixiviant $\mathrm{pH}$, rate of acidification, or both but did not change the growth rate (Fig. 2C and Supplementary Data 6). We re-arrayed disruption strains with differential acidification into new 96-well plates alongside proxy wild-type (pWT) strains 


\section{A. G. oxydans B58 Genome Annotation Statistics}

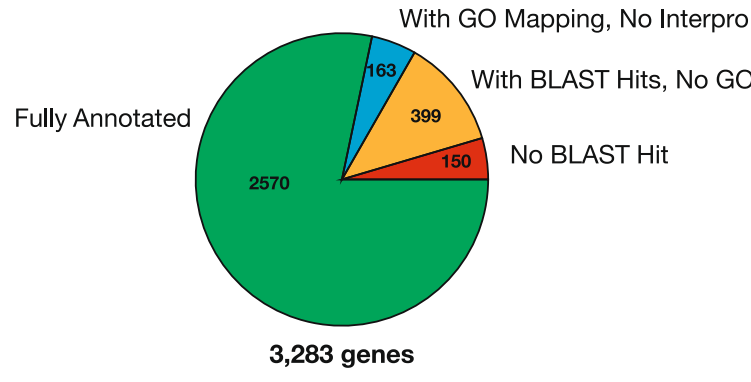

\section{GO enrichment for Non-disrupted Genes}

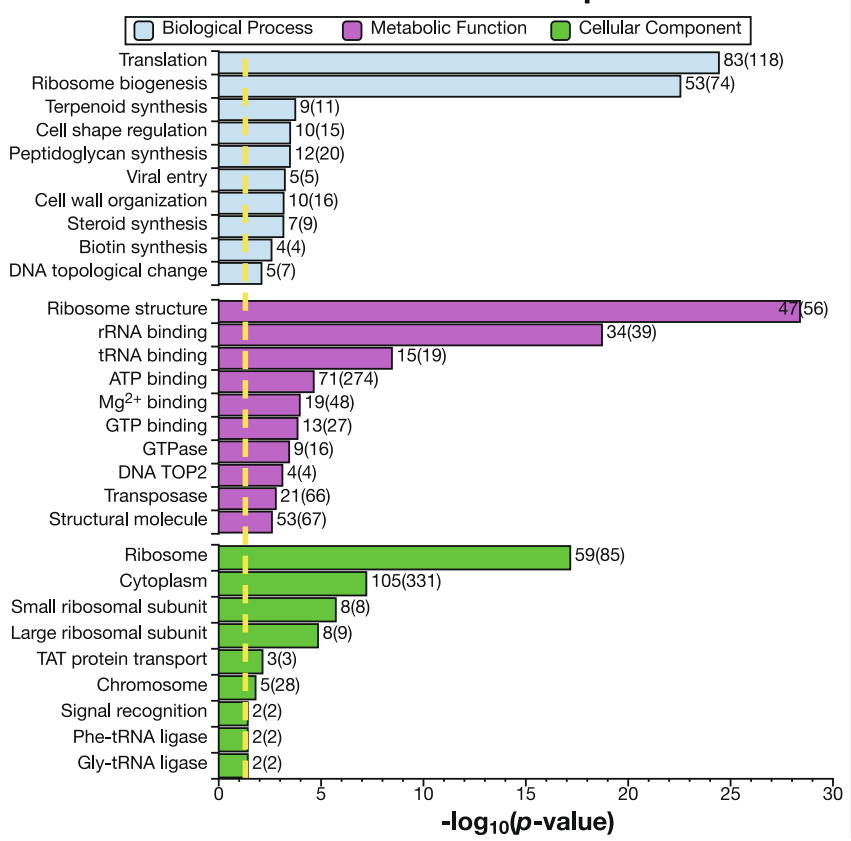

\section{B. Saturation of Possible Disruption Mutants}

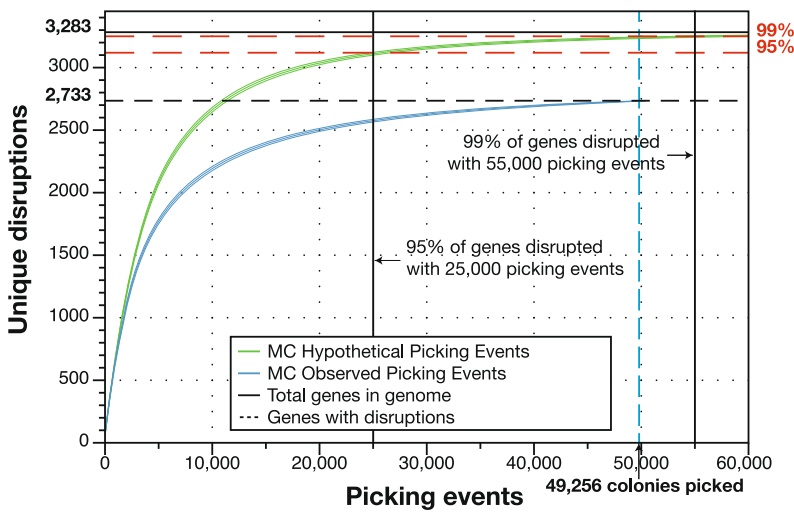

D. G. oxydans B58 Knockout Collection Statistics

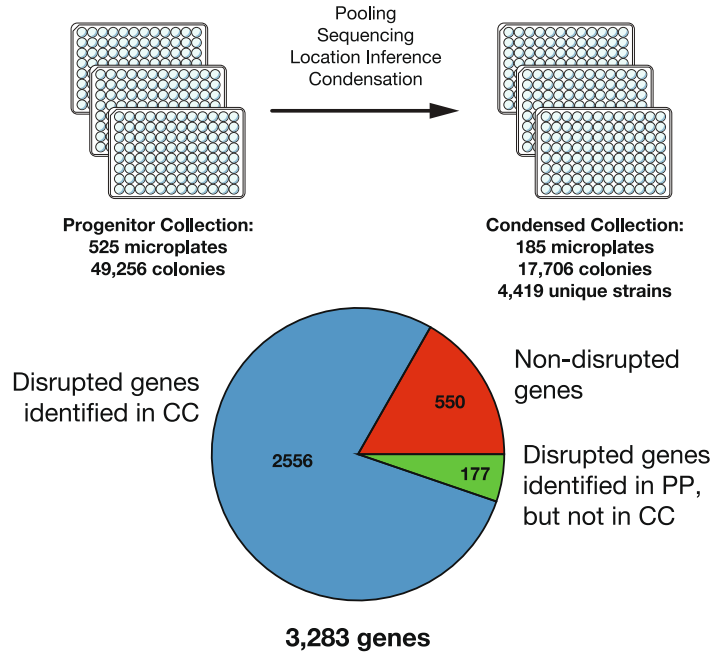

Fig. 1 Knockout Sudoku was used to curate a saturating coverage transposon insertion mutant collection for Gluconobacter oxydans B58. A The G. oxydans B58 genome contains 3283 genes. Two thousand five hundred and seventy genes were fully annotated with a BLAST hit, InterPro ID, and gene ontology (GO) group. An additional 163 genes had an annotation and GO group but lacked an InterPro ID; 399 retrieved only a BLAST hit, and 150 were unable to be assigned any annotation. B A Monte Carlo (MC) estimate (green curve) of the number of genes represented by at least one mutant as a function of the number of mutants collected demonstrated that picking 25,000 mutants would yield at least one disruption for $95 \%$ of genes, while picking 50,000 mutants would yield at least one disruption for $99 \%$ of genes. In total, we picked 49,256 single-gene disruption mutants and located at least one disruption for 2733 genes. A Monte Carlo simulation (blue curve) of picking with random drawing from the sequenced progenitor collection (PC) without replacements demonstrates that the genome coverage was truly saturated. The center of each curve is the mean value of the unique gene disruption count from 1000 simulations, while the upper and lower parts of each curve represent two standard deviations around this mean. C A one-sided Fisher's exact test for gene ontology enrichment among the non-disrupted (putatively essential) genes revealed significant enrichment $(p<0.05$, yellow line) of genes involved in translation and other ribosome-related functions. D The curated condensed collection (CC) contains 17,706 isolated colonies across 185 plates. High-throughput sequencing of the CC confirmed the location for 4419 unique disruption strains, representing disruptions in 2556 genes. Hundred and seventy-seven genes located in the PC were not located in the CC. No disruption mutant was detected in 550 genes.

(see "Methods" section) that have a transposon insertion in an intergenic region and show nondifferential growth or biolixiviant production (Supplementary Fig. 2). The new collection was reassayed with TB and BPB assays, and the strength and significance of each result were determined by comparison with pWT through a Bonferroni-corrected $t$-test (see "Methods" section). Mutants that cause the 25 largest reductions and 50 largest increases in endpoint acidity yet do not affect the growth rate are shown in Fig. 2D. A full set of mutants that cause significant changes in acidification are listed in Supplementary Data 6.

Thirty-one mutants that caused significant changes in the acidification rate without changing the growth rate are shown in Fig. 2E and Supplementary Data 6F. However, 14 of the faster strains, including $\delta G O \_868$, a disruption of a LacI-type transcriptional repressor, which was the fastest strain, produced a less acidic biolixiviant than the wild-type, indicating that targeting these genes for engineering a faster acidifier would likely be at the expense of a more acidic biolixiviant. None of the strains with a faster rate of acidification also created a more acidic biolixiviant. This result suggests that multiple genetic engineering interventions will be needed to construct a strain of G. oxydans that simultaneously produces a more acidic biolixiviant than the wild-type at a faster initial rate.

Pi transport and PQQ synthesis are main controllers of acidification. We used Fisher's exact test to determine which biological processes, metabolic functions, and cellular components are enriched 
A. TB Screen Finds Knockout of Gene Involved in Phosphate Transport Reduces Final Acidity

\section{B. BPB Screen Finds Knockout of Gene Likely Involved in PQQ Synthesis Slows Acidification Rate}

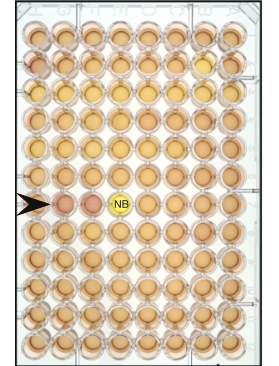

CC Plate 65

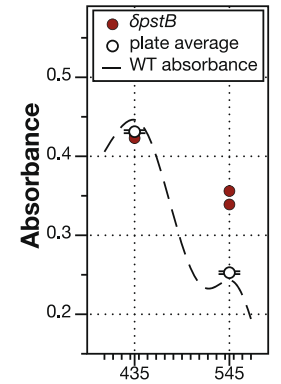

Wavelength

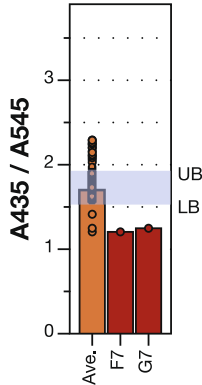

SpstB

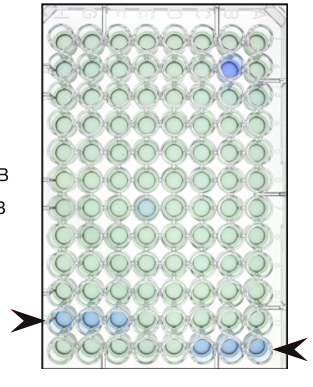

CC Plate 162

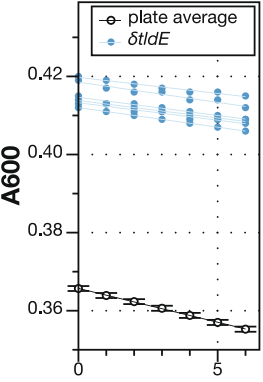

Time (minutes)

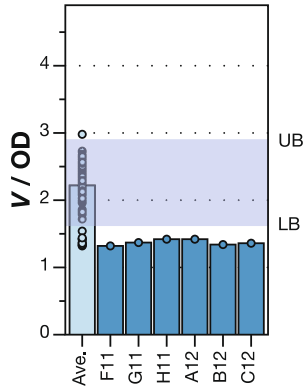

$\delta$ t/dE

C. Work flow for identifying comprehensive set of genes underlying acidification

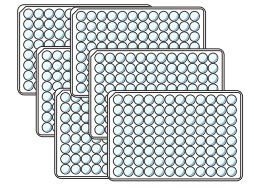

G. oxydans B58 Knockout Collection (185 microplates)

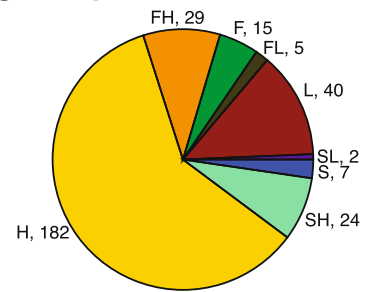

304 Total Screen Hits

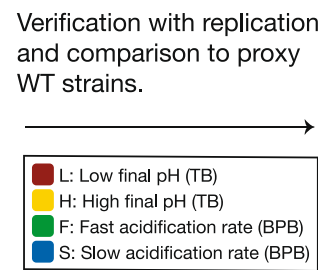

S: Slow acidification rate (BPB)

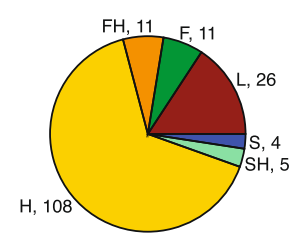

165 Total Verified Hits

D. 25 Biggest Reductions and 50 Biggest Increases in Biolixiviant Acidity

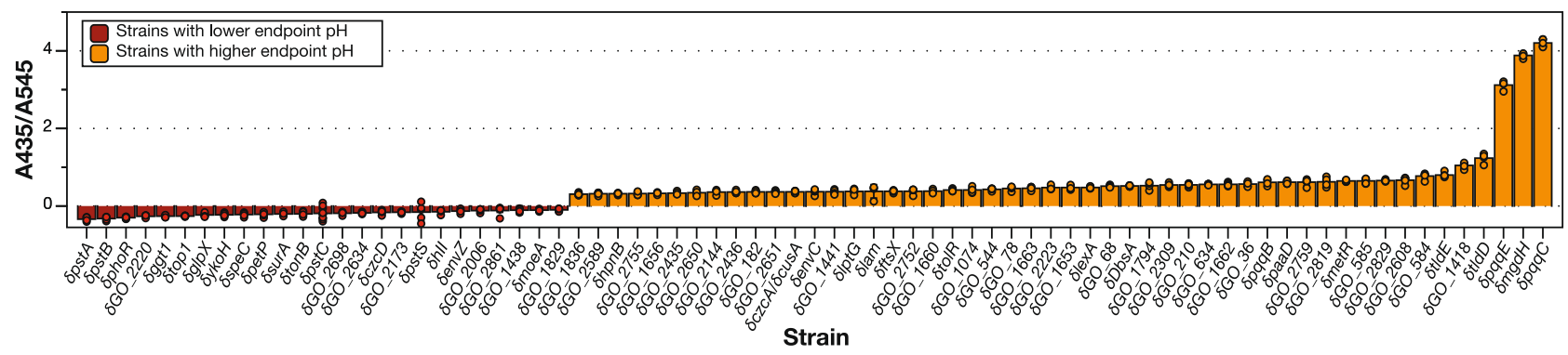

E. Most Significant Changes in Acidification Rate

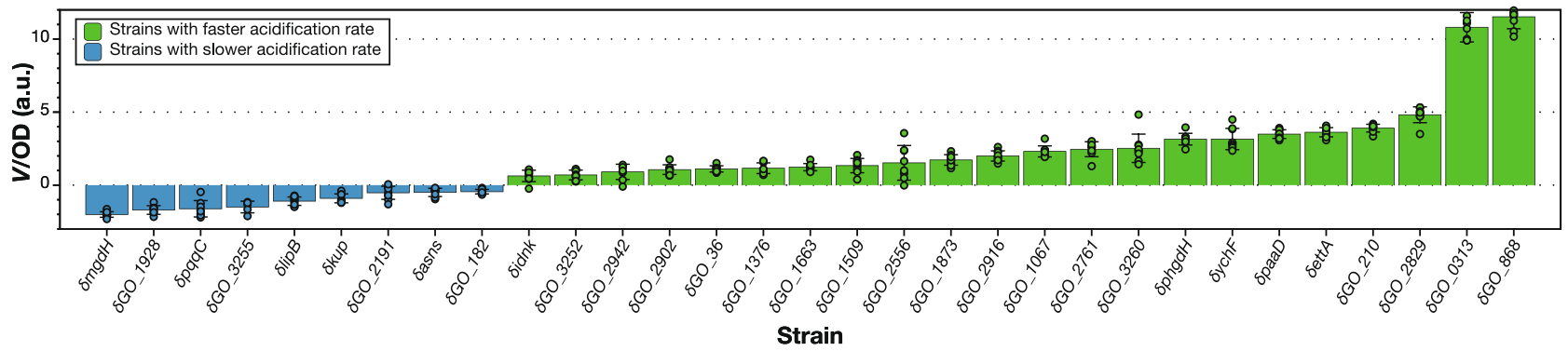

among the gene disruptions that significantly changed biolixiviant acidification (Fig. 3). Among the disrupted genes that led to a stronger acidity, the most significant enrichment for all three GO categories involves the phosphate-specific transport system, represented by $p s t A, p s t B, p s t C$, $p s t S$, and $p h o R$ (Fig. 3A). Other enriched ontologies include those related to phosphate signaling and binding.

Among the disrupted genes that led to weaker acidity, several enriched GO terms are related to the synthesis or use of the redox cofactor PQQ, represented by $p q q B, p q q C, p q q E, t l d D$, and $m g d h$ (Fig. 3B). Other enriched ontologies include those related to carbohydrate metabolism, represented by five enzymes related to fructose metabolism: Fructokinase (GO_1072), GDP-mannose 4,6-dehydratase (GO_1441), Mannose-1-phosphate guanylyl transferase/Mannose-6-phosphate isomerase (GO_182),
UTP--glucose-1-phosphate uridylyltransferase /Phosphomannomutase (GO_1923), and UDP-glucose 4-epimerase (GO_36) (Supplementary Data 2C).

Acidification rate is controlled by carbohydrate metabolism and respiration. Disruptions in the pentose phosphate pathway and related metabolic processes increased the acidification rate (Fig. 3C). Meanwhile, disruptions of the electron transport pathway components are the most significantly enriched group of mutants that decreased the acidification rate (Fig. 3D).

Dye assay results are validated by direct $\mathbf{p H}$ measurements. We selected 22 strains from those with the greatest increase or decrease in acidification (Fig. 2D, E) for further testing. Dye $\mathrm{pH}$ measurements were validated by direct $\mathrm{pH}$ measurements. Eleven 
Fig. 2 High-throughput pH screens of the G. oxydans whole genome knockout collection were used to identify genes that control REE bioleaching. A Thymol blue (TB) was used to measure the endpoint acidity of biolixiviant produced by each well of the condensed collection. The ratio of TB absorbance (A) at 435 and $545 \mathrm{~nm}$ is linearly related to $\mathrm{pH}$ between 2 and 3.4 (Supplementary Fig. 1). CC plate 65 contains biolixiviant produced by $\delta p s t B$ strain in wells F7 and G7 (arrowhead), whose absorbance at 435 and $545 \mathrm{~nm}$ are shown (middle panel, red dots), along with the average absorbance of all occupied wells on the plate $(n=94)$ (white dot; error bars are SEM). The dashed line represents a typical absorbance spectrum for WT-produced biolixiviant. The A435/A545 ratio for these two wells (right panel, red bars and dots) compared with the mean ratio of the plate (orange bar; orange dots show individual data points) is well below the lower bound (LB) for the plate, indicating that $\delta p s t B$ produces a much more acidic biolixiviant than the average strain. B Bromophenol blue (BPB) was used to measure rate of change in $\mathrm{pH}$ at the onset of glucose conversion to organic acids. Rate was measured over a 6 min period within five minutes of adding bacteria to a glucose and BPB solution. Condensed collection (CC) plate 162 contains the $\delta$ tldE strain in wells F11-C12 (arrowheads), whose changes in absorbance over time are graphed along with the average for that plate (middle panel, $n=94$ ). A comparison of the normalized rate over OD for each well (right panel, dark blue bars and dots) versus the plate mean (light blue bar; dots show individual data points) shows how V/OD for these wells was below the lower bound for CC plate 162. C All 185 plates of the CC were screened for acidification using TB and BPB assays. Hits from both screens were verified in comparison with proxy WT strains. In total, 176 disruption strains were shown to significantly contribute to acidification. D, E Comparisons with proxy WT strains were made by a two-tailed $t$-test with a Bonferroni-corrected alpha $(\alpha=0.05 / N$ where $N$ is the number of comparisons). Bars represent mean values and dots represent individual data points. Error bars represent standard deviation. D The 25 largest significant reductions in biolixiviant $\mathrm{pH}$ and 50 largest significant increases in biolixiviant $\mathrm{pH}$. $\mathrm{N}=120$ or $\mathrm{N}=242$ for comparisons with $\mathrm{pWT}$ set $\mathrm{A}$ or set $\mathrm{B}$, respectively. (The full data set and number of biological replicates for each strain can be found in Supplementary Data $6 \mathrm{E}$ ). E All significant changes in acidification rate, $N=60$ (The full data set and number of biological replicates for each strain can be found in Supplementary Data 6F).
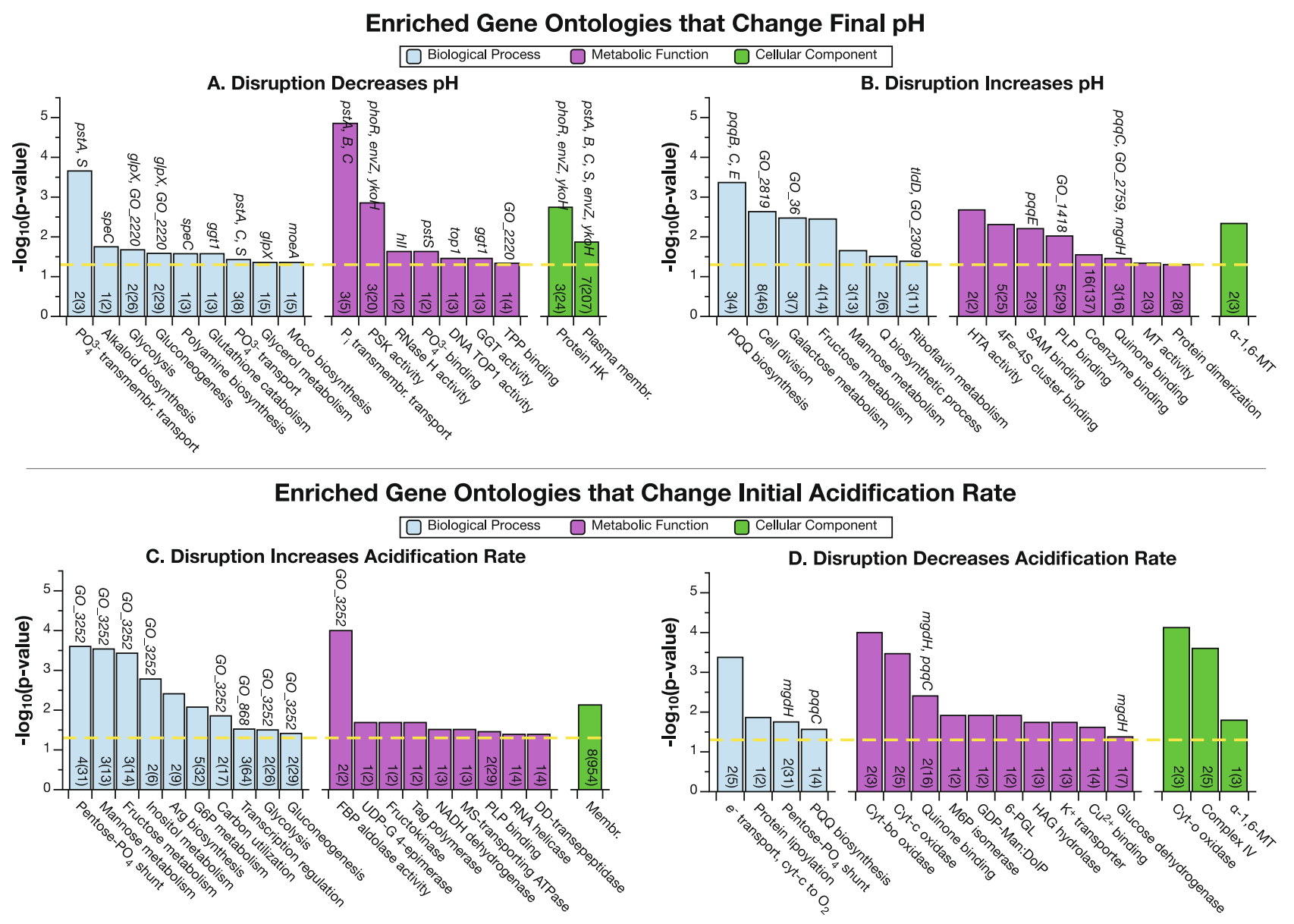

Fig. 3 Genes involved in phosphate signaling, carbohydrate metabolism and PQQ synthesis were significantly overrepresented in the significant hits from high-throughput screens of acidification by G. oxydans. A one-sided Fisher's Exact Test was used to test for gene ontology enrichment $(p<0.05$, yellow dashed line). Numbers at the base of bars are how many genes from the significant hits are from that gene ontology (GO), out of the total in the genome (in parentheses). Genes selected for further analysis of endpoint pH and bioleaching (Fig. 4) that contribute to an enriched GO are listed above the bars. A, B Enriched GO terms among genes that decreased and increased the end point pH. C, D Enriched GO terms among genes that increased and decreased the initial acidification rate. FBP fructose-bisphosphate, GDP-Man:DolP dolichyl-phosphate beta-D-mannosyltransferase, GGT glutathione hydrolase, G6P glucose 6phosphate, HTA homoserine O-acetyltransferase, DD-transepeptidase D-Ala-D-Ala carboxypeptidase, HAG hydroxyacylglutathione, Membr membrane, Moco Mo-molybdopterin cofactor, MS monosaccharide, MT mannosyltransferase, M6P mannose-6-phosphate, Pi inorgan ${ }_{i}$ c phosphate, PLP pyridoxal phosphate, PQQ pyrroloquinoline quinone, PSK phosphorelay sensor kinase, Q queuosine, RNase H DNA-RNA hybrid ribonuclease, SAM S-adenosyl-L-methionine, TPP thiamine pyrophosphate, TOP1 topoisomerase type 1, HK histidine kinase, UDP-G uracil-diphosphate glucose, 6-PGL 6-phosphogluconolactonase. 

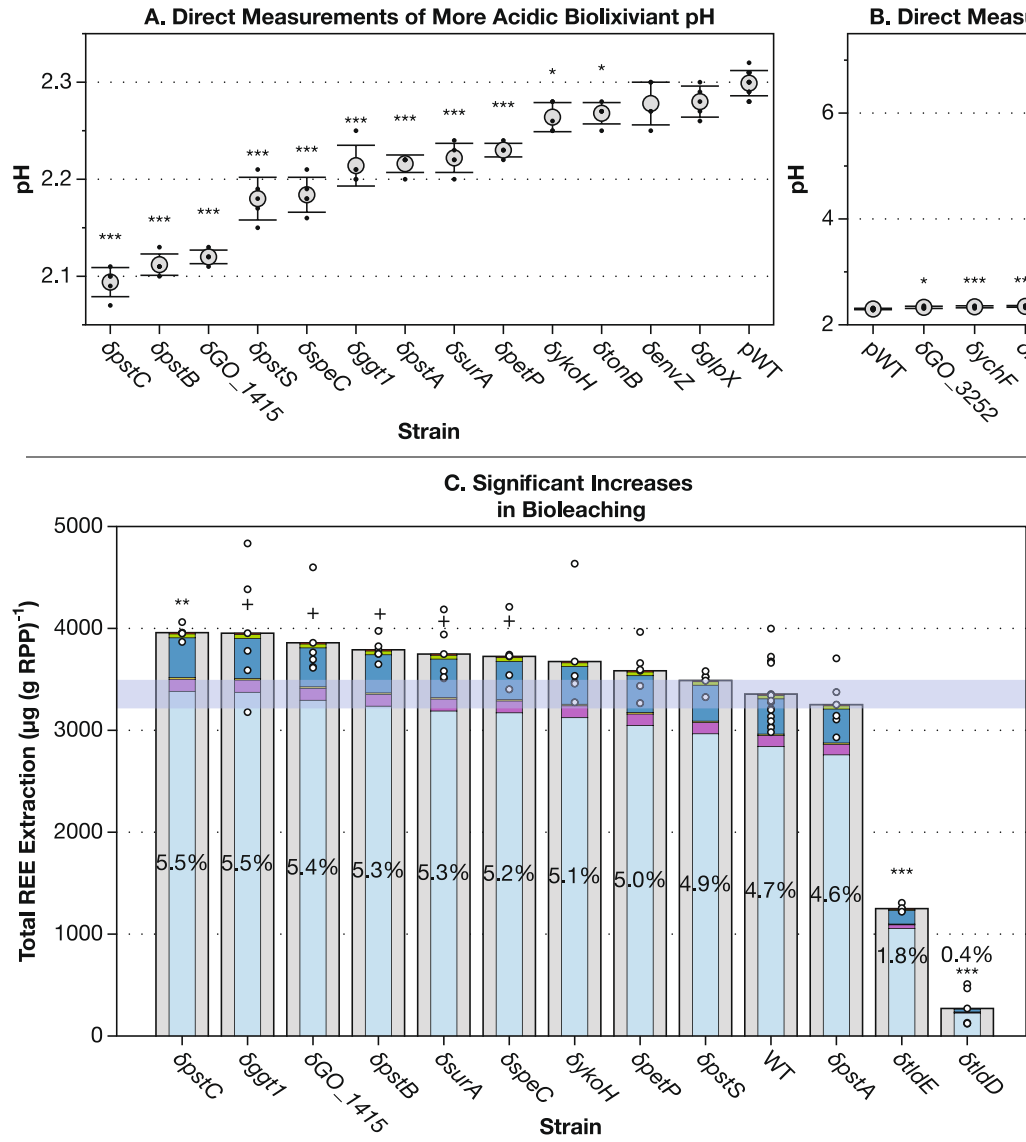

B. Direct Measurements of Less Acidic Biolixiviant pH

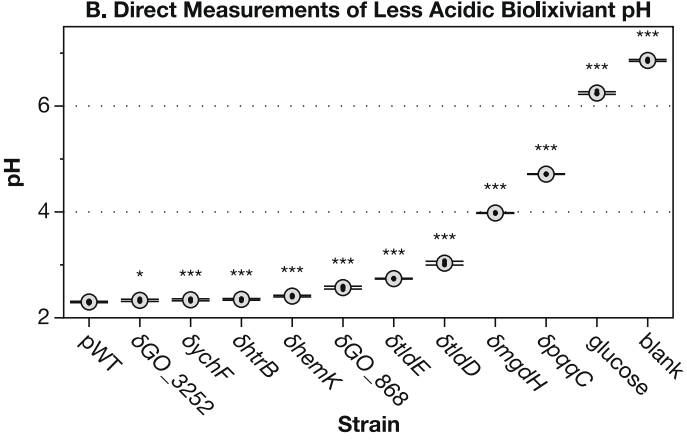

Strain
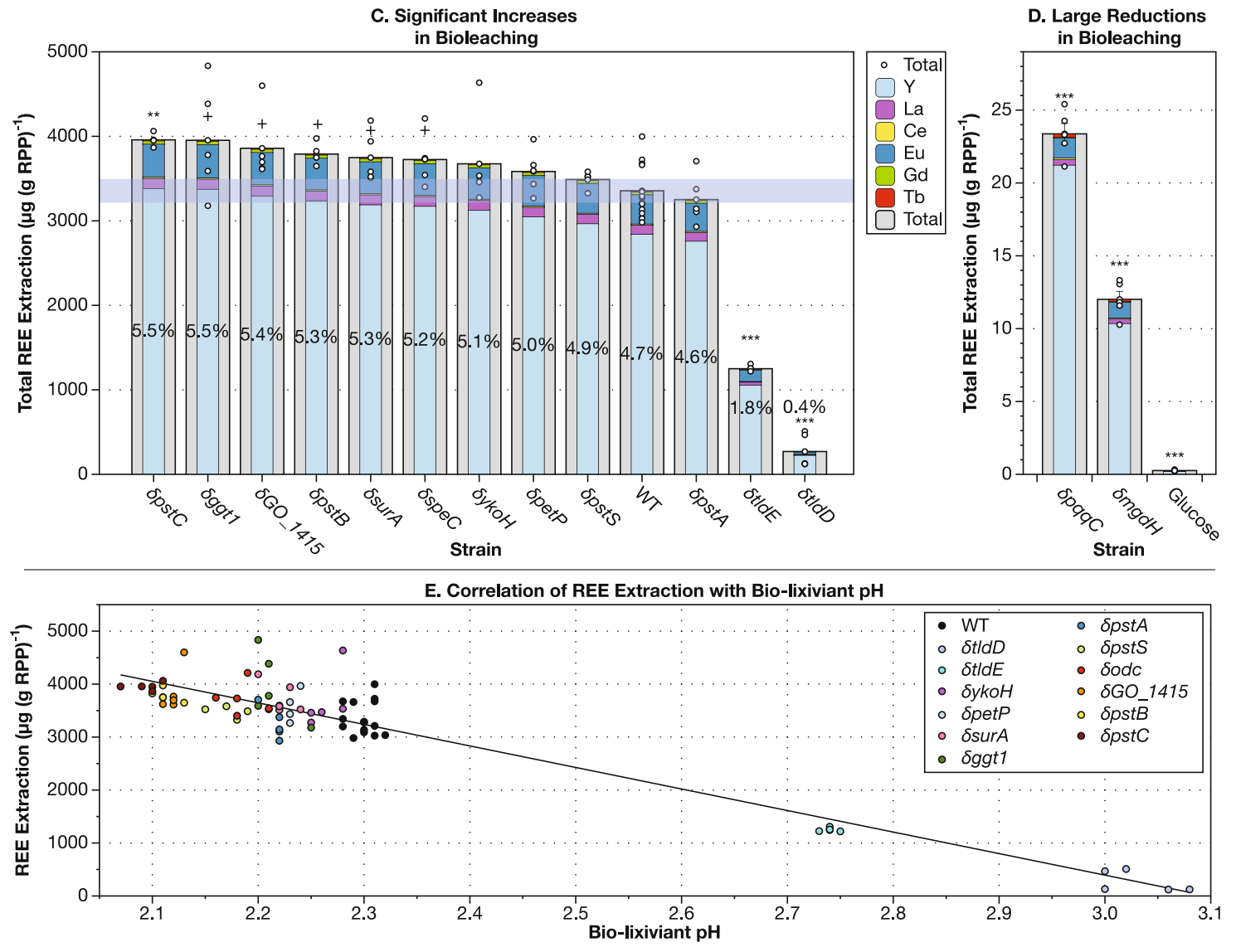

Fig. 4 Increased acidification strains of G. oxydans B58 are able to increase rare earth extraction from retorted phosphor powder (RPP). A, B A subset of 22 disruption strains was tested for acidification with direct $\mathrm{pH}$ measurement. Gray circles represent the mean $\mathrm{pH}$ for each strain and comparisons were made with wild type by two-tailed $t$-test with Bonferonni correction $(N=22)$. $\mathrm{pH}$ measurements significantly different from $\mathrm{pWT}$ are labeled with asterisks: ${ }^{\star} p<0.05 / 22$; ${ }^{\star \star} p<0.01 / 22 ;{ }^{\star \star \star} p<0.001 / 22(n=5$ biological replicates, $\mathrm{df}=18)$. Error bars represent standard deviation. Individual data points are shown as black dots.

C, $\mathbf{D}$ Ten disruption strains with the lowest final biolixiviant $\mathrm{pH}$ and four with the highest were tested for RPP bioleaching capabilities. Outer gray bars represent the mean total REE extracted for each biolixiviant. Inner multicolored bars represent fractional contributions of each REE. Error bars represent standard error around the mean total REE extracted. Percentages are total REE extraction efficiency (based on previously published REE amounts in the RPP26). Individual data points for total REE extracted are shown as white dots. C Comparison with a two-tailed $t$-test between each mutant and pWT demonstrated that eight strains were significantly better or worse at bioleaching total REEs $(+p<0.05 ; n=5$ biological replicates, $\mathrm{df}=18)$. With a Bonferroni correction $(N=12)$, only one was significantly better ${ }^{* \star} p=5.30 \times 10^{-4}$ for $\left.\delta p s t C\right)$, but two of the higher $\mathrm{pH}$ biolixiviants that extracted detectable REEs were significantly attenuated in bioleaching capability ${ }^{\star \star \star} p=1.71 \times 10^{-11}$ and $6.32 \times 10^{-14}$ for $\delta$ tldE and $\delta t / d D$, respectively). Light purple shading represents the mean and standard deviation for total REE bioleached by pWT biolixiviant. D Disruption mutants for mgdh and pqqC are only able to extract less than $1 \%$ of the REE that wild-type G. oxydans can but still extract significantly more REE than glucose alone when measured at a lesser dilution (two-tailed $t$-test with Bonferroni correction, $N=2:^{\star \star \star} p=9.71 \times 10^{-10}$ and $2.47 \times 10^{-8}$ for $\delta p q q C$ and $\delta m g d h$, respectively, $n=5$ biological replicates). E Total REE extraction is plotted versus $\mathrm{pH}$ for each replicate for each strain, demonstrating how the two are linearly related. Each data point is color coded by strain, as indicated in the plot legend.

of the 13 strains that produced significantly lower acidity biolixiviants in TB assays did the same in direct $\mathrm{pH}$ measurements (Fig. 4A). The most acidic biolixiviant was produced by a disruption in the phosphate transport gene $\delta p s t C$ at $\mathrm{pH} 2.09$. In fact, 4 of the 11 mutant strains that produced a more acidic biolixiviant were disrupted in genes involved in the phosphatespecific transport system ( $\delta p s t A, \delta p s t B, \delta p s t C$, and $\delta p s t S)$.

Additional disruptions that led to a more acidic biolixiviant included those in a hypothetical protein with no similarity to anything previously characterized (SGO_1415); a 
gamma-glutamyltranspeptidase ( $\delta g g t 1)$; a periplasmic chaperone $(\delta s u r A)$; an HTH-type transcriptional regulator $(\delta p e t P)$; a two-component system sensor histidine kinase $(\delta y k o H)$; a pyridoxal 5-phosphate (PLP)-dependent ornithine decarboxylase $(\delta s p e C)$; and a TPR domain protein that is a putative component of the TonB iron uptake system $(\delta \operatorname{ton} B)$.

Nine of the tested strains produced biolixiviants significantly higher in $\mathrm{pH}$ than pWT (Fig. 4B). The most alkaline biolixiviant was produced by a disruption in the PQQ synthesis system, $\delta p q q C$, at $\mathrm{pH} 4.71$. While $\delta p q q C$ produced very little acid, this is below the $\mathrm{pH}$ of glucose in media alone, indicating that some bacterial acidification still occurred. In fact, three of the nine mutants that produce biolixiviants with reduced acidity either synthesize PQQ ( $\delta p q q C$ and $\delta t l d D)$ or use it as a cofactor

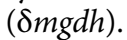

Additional disruptions that led to a more alkaline biolixiviant than pWT include a fructose-bisphosphate aldolase class II ( $\left.\delta G O \_3252\right) ;$ a GTP and nucleic acid binding protein $(\delta y c h F)$; a lipid A biosynthesis protein $(\delta h t r B)$; a peptide chain release factor (ShemK); the LacI-type transcriptional repressor that increases the initial acidification rate (8GO_868); and the second component of a proteolytic complex with TldD $(\delta t l d E)$.

Disrupting the phosphate transport system increases bioleaching. We tested whether ten of the mutants that produced a more acidic biolixiviant could bioleach REE from retorted phosphor powder (RPP) from spent fluorescent lightbulbs more efficiently than pWT (Fig. 4C). For each mutant, the elemental composition of REE leachate was similar to that previously reported ${ }^{26}$. Six of these mutants significantly increased bioleaching. Two of the better bioleaching mutants disrupted the $p s t$ phosphate transport system $(\delta p s t C$ and $\delta p s t B)$. Overall, we found that bioleaching efficiency correlates with biolixiviant $\mathrm{pH}$, as expected (Fig. 4E).

The $\delta p s t C$ mutant produced the most acidic biolixivant and extracted the most REE from RPP: 5.5\% total extraction efficiency compared with $\mathrm{pWT}(4.7 \%)$. In other words, $\delta p s t C$ removed $18 \%$ more REEs from RPP than pWT. This increase in REE extraction remains significant even after adjusting the a to account for the possibility of a difference due to chance alone (Bonferroni correction, see "Methods" section). Without the adjustment, six of the better acidifiers were also better bioleachers than pWT (Fig. 4C). The remaining better bioleachers increased REE extraction by between $11 \%(\delta s p e C)$ and $18 \%$ ( $\delta g g t 1)$ (Fig. 4C).

We speculate that disrupting phosphate transport and signaling derepresses acid production in G. oxydans. Six of the disruption strains that resulted in a lower biolixiviant $\mathrm{pH}(\delta p s t C$, $\delta p s t B, \delta g g t 1, \delta p s t A, \delta p s t S$, and $\delta y k o H)$, including three that increased bioleaching $(\delta p s t C, \delta p s t B, \delta g g t 1)$, along with many more identified by acidification high-throughput assays (e.g., $\delta p h o R, \delta e n v Z$ ), are involved in phosphate transport, sensing and signaling.

In its natural environment, G. oxydans produces biolixiviants to liberate phosphate from minerals, not metals ${ }^{38-40}$. Under phosphate-limiting conditions, the PstSCAB phosphate transporter will activate the histidine kinase PhoR, which in turn phosphorylates the transcription factor PhoB and activates the pho regulon, enabling phosphate solubilization and uptake ${ }^{41}$. Under sufficient phosphate conditions, PhoB is deactivated by PhoR, which in turn inhibits the expression of genes involved in the phosphate-starvation response. We speculate that by disrupting pstSCAB or PhoR, we prevent G. oxydans from sensing when there is adequate phosphate in its environment and when to stop producing biolixiviants.
Disrupting mgdh and PQQ synthesis genes decreases bioleaching. We also tested REE extraction by four mutants that produce a less acidic biolixiviant than $\mathrm{pWT}$, and all were worse bioleachers than pWT (Fig. 4C, D). Unsurprisingly, the $\delta m g d h$ mutant was the worst bioleacher of all tested mutants, considering its lack of gluconic acid production ${ }^{35}$. $\delta m g d h$ reduced bioleaching by $97 \%$. Disruption mutants that knocked out the synthesis of mGDH's essential redox cofactor, PQQ, also produced significant reductions in biolixiviant acidity. $\delta p q q C$ reduced bioleaching by $\approx 94 \%$. While bioleaching by $\delta m g d h$ and $\delta p q q C$ was negligible compared to $\mathrm{pWT}$, they were able to bioleach a statistically significant amount of REE compared to glucose alone. This indicates, as previously speculated ${ }^{26}$, that a bioleaching mechanism independent of $\mathrm{mGDH}$ exists in $G$. oxydans (Fig. 4D).

Disruption mutants in $t l d D$ and $t l d E$ were also much worse at bioleaching than pWT. $\delta t l d D$ reduces bioleaching by $92 \%$, while $\delta$ tldE reduces it by $63 \%$ (Fig. $4 \mathrm{C}$ ). We speculate that TldD and TldE contribute to the supply of the PQQ cofactor to mGDH. $\delta t l d D$ strongly attenuates acid production (Fig. $4 \mathrm{~B}$ ), and the gene has already been implicated in PQQ synthesis in $G$. oxydans $621 \mathrm{H}^{42}$. In E. coli, TldD and TldE form a two-component protease for the final cleavage step in the processing of the peptide antibiotic microcin $\mathrm{B} 17^{43}$. In a similar manner, PqqF and PqqG from Methylorubrum extorquens form a protease that rapidly cleaves PqqA, the peptide precursor to $\mathrm{PQQ}^{44}$. We speculate that TldD in G. oxydans plays the same role as PqqF from $M$. extorquens, while TldE plays the same role as PqqG. Deletion of pqqF in $M$. extorquens completely inhibits cleavage of PqqA, while we found that disruption of tldD in G. oxydans reduces REE bioleaching by $92 \%$. Moreover, deletion of $p q q G$ in $M$. extorquens only reduces PqqA cleavage by $50 \%{ }^{44}$, while disruption of $t l d E$ only reduces REE bioleaching by $63 \%$. These parallels strongly indicate a role for TldE in the biosynthesis of PQQ in G. oxydans.

\section{Discussion}

Bioleaching has the potential to revolutionize the environmental impact of REE production and dramatically increase access to these critical ingredients for sustainable energy technology. However, making REE bioleaching cost competitive with thermochemical methods will require increasing both the rate and completeness (overall efficiency) of REE extraction. This work provides a roadmap for improving bioleaching by genetic engineering.

By constructing a whole genome knockout collection for $G$. oxydans, one of the most promising organisms for REE bioleaching, we are able to characterize the genetics of this process with high sensitivity and high completeness. In total, we identified 165 gene disruption mutants that significantly changed the acidity of its biolixiviant, rate of production, or both. The regulatory elements of each of these genes represent dials that can be turned to improve bioleaching.

As well as producing gluconic acid, G. oxydans can be used in the production of industrially important products, including 2,5-diketogluconic acid, a precursor of vitamin $\mathrm{C}_{;}^{45} ; 5$-ketogluconic acid, a precursor of $\mathrm{L}(+)$-tartaric acid used in the production of food and pharmaceuticals ${ }^{45}$, vinegar, sorbitol, and dihydroxyaceton $e^{45}$. The G. oxydans knockout collection will allow characterization of the production of these metabolites and improvement of their production.

REE bioleaching by G. oxydans is predominantly controlled by two well-characterized systems: phosphate signaling and incomplete glucose oxidation, which is supported by the production of the redox cofactor PQQ. Interrupting phosphate signaling control of biolixiviant production by disrupting a single gene $(p s t C)$ can 
increase REE extraction by $18 \%$. Disrupting the supply of the PQQ cofactor to the membrane-bound glucose dehydrogenase reduces REE extraction by up to $94 \%$.

Among the many genes whose disruption attenuated final biolixiviant acidity, several were related to fructose metabolism. $G$. oxydans converts mannitol (the carbon source used for growth in these studies) into fructose, which can be incorporated into the pentose phosphate and Enter-Douderoff pathways, the latter of which can result in accumulation of acetate in the growth media ${ }^{46}$. As these five disruptions did not affect growth on mannitol, the genes are likely dispensable for fructose catabolism, but may be important for acetate accumulation and some initial media acidification during growth on mannitol. Conversely, disruptions in other genes related to carbohydrate metabolism conferred a faster rate of acidification, most likely due to the disruption of glucose catabolism/incorporation into the pentose phosphate pathway and a preference for incomplete oxidation and gluconic acid production.

Comprehensive screening of the G. oxydans genome also revealed targets that could not have been predicted to contribute to REE bioleaching. For example, disrupting GO_1415, encoding a protein of unknown function, increases REE bioleaching by $15 \%$. Additionally, our results highlight the potential for a previously uncharacterized role of TldE in PQQ synthesis. Several periplasmic dehydrogenases in G. oxydans depend on PQQ for their function ${ }^{47}$, and in the case of the D-sorbitol dehydrogenase, mSLDH, overexpression of the $p q q$ synthase genes and tldD enhances conversion of N-2-hydroxyethylglucamine into 6-(N-hydroxyethyl)-amino-6-deoxy-L-sorbofuranose (6NSL), a precursor to the diabetes drug Miglitol ${ }^{48,49}$. The discovery of the potential contribution of TldE to PQQ biosynthesis may allow for exceptional enhancement of cofactor production through the additional overexpression of this gene and a consequent uptick in dehydrogenase activity. PQQ is an essential cofactor important for several other industrial applications of G. oxydans, including the production of L-sorbose $\mathrm{e}^{50}$ and 5-keto-D-gluconate ${ }^{51}$. Furthermore, PQQ alone has many applications across many biological processes, from plant protection to neuron regeneration ${ }^{52}$.

Our results demonstrate the potential for improving bioleaching through genetic engineering. Furthermore, the creation of a whole-genome knockout collection in G. oxydans will facilitate its use as a model species for further studies in REE bioleaching and other industrially important applications of similar acetic acid bacteria. The findings of the two major systems contributing to acidification in G. oxydans suggest the first steps in the roadmap for greatly improving bioleaching: take the brakes off regulation of acid production by disabling the phosphatespecific transport system, while overexpressing $m g d h$ along with the expanded synthesis pathway for its cofactor PQQ.

\section{Methods}

Gluconobacter oxydans B58 genome sequencing. Gluconobacter oxydans strain NRRL B-58 (GoB58) was obtained from the American Type Culture Collection (ATTC), Manassas, VA. In all experiments, GoB58 was cultured in yeast peptone mannitol media [YPM; $5 \mathrm{~g} \mathrm{~L}^{-1}$ yeast extract (C7341, Hardy Diagnostics, Santa Maria, CA), $3 \mathrm{~g} \mathrm{~L}^{-1}$ peptone (211677, BD, Franklin Lakes, NJ), $25 \mathrm{~g} \mathrm{~L}^{-1}$ mannitol (BDH9248, VWR Chemicals, Radnor, PA)] with or without antibiotic, as specified. Genomic DNA was extracted from saturated culture using a Quick-DNA Miniprep kit from Zymo Research (Part number D3024, Irvine, CA). A genomic DNA library was prepared and sequenced using a TruSeq DNA PCR-Free Library Prep Kit (Illumina, San Diego, CA).

The prepared library was sequenced on a MiSeq Nano (Illumina, San Diego, CA, USA) with a $500 \mathrm{bp}$ kit at the Cornell University Institute of Biotechnology (Ithaca, NY, USA). The resulting paired-end reads were trimmed using Trimmomatic ${ }^{53}$ and assembled with SPAdes using k-mer sizes 21, 33, 55, 77, 99, and 127 and an auto coverage cutoff ${ }^{4}$. Assembly quality was checked with QUAST ${ }^{55}$, and genome completeness was verified with BUSCO ${ }^{56}$ using the proteobacteria_odb9 database (available for download at https://datacommons.cyverse.org/browse/iplant/home/ shared/iplantcollaborative/example_data/BUSCO.sample.data/proteobacteria_odb9/ ancestral) for comparison. The resulting 62 contigs were annotated online using RAST (https://rast.nmpdr.org) ${ }^{57-59}$.
Gene ontology enrichment. DIAMOND ${ }^{60}$ was used to assign annotated protein models with the closest BLAST hit using the Uniref90 database (available for download at https://www.uniprot.org/uniref/), an $E$-value threshold of $10^{-10}$, and a block size of 10 . InterProScan ${ }^{61}$ (version 5.50-84.0) was used to assign family and domain information to protein models.

Output from both of these searches was used to assign gene ontologies with BLAST2GO ${ }^{62}$. Gene set enrichment analysis was performed with the BioConductor topGO package ${ }^{63}$ using the default weight algorithm, the TopGO Fisher test, with a $p$-value threshold of 0.05 .

Mating for transposon insertional mutagenesis. The transposon insertion plasmid pMiniHimarFRT ${ }^{36}$ was delivered to GoB58 by conjugation with E. coli WM3064. E. coli WM3064 transformed with pMiniHimarFRT was grown overnight to saturation in $50 \mathrm{~mL} \mathrm{LB}\left(10 \mathrm{~g} \mathrm{~L}^{-1}\right.$ tryptone, $5 \mathrm{~g} \mathrm{~L}^{-1}$ yeast extract, and $\left.10 \mathrm{~g} \mathrm{~L}^{-1} \mathrm{NaCl}\right)$ supplemented with $50 \mu \mathrm{g} \mathrm{mL}^{-1}$ kanamycin (kan) and $90 \mu \mathrm{M}$ diaminopimelic acid (DAP; D1377, Sigma-Aldrich, St. Louis, MO), rinsed once with $50 \mathrm{~mL} \mathrm{LB}$, and then resuspended in $20 \mathrm{~mL}$ YPM.

GoB58 was grown for approximately $24 \mathrm{~h}$ in YPM, diluted to an optical density (OD) of 0.05 in $750 \mathrm{~mL}$ YPM and incubated at $30^{\circ} \mathrm{C}$ for two doublings until the OD reached 0.2 . GoB58 culture was distributed into $1350 \mathrm{~mL}$ conical tubes, to which rinsed and resuspended WM3064 was added at a ratio of 1:1 by density (approximately $1 \mathrm{~mL}$ WM3064 to $50 \mathrm{~mL}$ B58). Bacteria were mixed by inversion and then centrifuged at $1900 \times g$ for $5 \mathrm{~min}$. The supernatant was poured off, and the mixture was resuspended in the remaining liquid $(\approx 0.5 \mathrm{~mL})$, dispensed onto a YPM agar petri dish in five spots of $0.1 \mathrm{~mL}$, and allowed to dry on the bench under a flame.

Mating plates were incubated at $30^{\circ} \mathrm{C}$ for $24 \mathrm{~h}$. Mating spots were collected by adding $4 \mathrm{~mL}$ YPM to a plate, scraping the spots into the liquid, and then suspending bacteria by drawing the liquid up and down several times with a pipette. Suspended cells were collected from each plate, and the suspension was distributed onto petri dishes with YPM agar supplemented with $100 \mu \mathrm{g} \mathrm{mL}^{-1}$ kanamycin at $100 \mu \mathrm{L}$ per dish.

After 3 days of incubation at $30^{\circ} \mathrm{C}$, colonies were transferred into 96-well microplates using a CP7200 colony picking robot (Norgren Systems, Ronceverte $\mathrm{WV}, \mathrm{USA})$. Each well contained $150 \mu \mathrm{L}$ YPM with $100 \mu \mathrm{g} \mathrm{mL}^{-1}$ kanamycin. For all high-throughput experiments, GoB58 was grown in polypropylene microplates sealed with a sterile porous membrane (Aeraseal, Catalog Number BS-25, Excel Scientific) and incubated at $30^{\circ} \mathrm{C}$ with shaking at $800 \mathrm{rpm}$. Isolated disruption strains were grown for three days to allow cultures in all wells to reach saturated growth. Wells B2 and E7 of each plate were reserved as no-bacteria controls.

A Monte Carlo numerical simulation (collectionm $\mathrm{c}^{36}$ ) was used to approximate how many insertions would need to occur before a mutant is found representing a knockout of each gene in the genome, which demonstrated that approximately 55,000 mutants would need to be generated and selected to identify mutants in at least $99 \%$ of all GoB58 genes (Fig. 1B).

In total, 18 matings were required to recover and transfer a progenitor collection of 49,256 disruption strains into 525 microplates over the course of approximately two months. Microplates with saturated cultures were maintained at $4^{\circ} \mathrm{C}$ for up to 3 weeks and incubated an additional night at $30^{\circ} \mathrm{C}$ before pooling. The progenitor collection was cataloged using the Knockout Sudoku combinatorial pooling method. Combinatorial pooling was done in three batches. The 525 plates were virtually arranged in a 20 by 27 grid, and creation of the progenitor collection catalog by combinatorial pooling, cryopreservation, pool amplicon library generation, and massively parallel sequencing were all performed using the protocol described by Anzai et al. ${ }^{36,37}$.

Curation of a whole-genome knockout collection. The condensed G. oxydans knockout collection was curated by manual curation of the progenitor collection catalog (as opposed to automatic curation in the Knockout Sudoku protocol ${ }^{36,37}$ ). To create a condensed collection, a disruption strain was chosen for each of the 2733 disrupted genes available in the progenitor collection, first prioritizing close proximity to the translation start and then the total probability of the proposed progenitor collection address. A second strain was chosen from the remaining strains for each gene that had another available. For 50 genes, the locations for both disruption strains selected were ambiguous, and thus, a third strain was selected from the remaining collection.

In total, 5137 disruption strains were transferred for isolation of single colonies. Many progenitor wells were predicted to have more than one possible strain per well, so for each strain, the number of colonies isolated was two times the predicted number of strains in the progenitor well, up to ten. The condensed collection, which amounted to 17,706 wells, was pooled, sequenced, and validated using the validation procedures detailed in the Knockout Sudoku protocol ${ }^{36,37}$. Unknown disruption strains significantly linked to acidification were identified with Sanger sequencing, also as previously described, with the exception of transposon-specific primers. For the first and second rounds of nested PCR, the transposon-specific primers were (5'GTATCGCCGCTCCCG-3', and (5'-CATCGCCTTCTATCGCCTTC-3), respectively.

Thymol blue endpoint acidity assay. Endpoint acidity was measured using the $\mathrm{pH}$ indicator thymol blue (TB; SKU114545, Sigma-Aldrich, St. Louis, MO), which changes from red to yellow below a $\mathrm{pH}$ of 2.8 (https://www.sigmaaldrich.com/US/ 
en/product/sial/114545). The lowest $\mathrm{pH}$ of biolixiviant generated by GoB58 was $2.3^{26}$; thus, TB allows for distinguishing strains that lower the $\mathrm{pH}$ below that of the wild-type biolixiviant. To generate biolixiviants, the condensed collection was replicated by transferring on pins from cryopreserved culture plates into new growth plates containing $100 \mu \mathrm{L}$ YPM with $100 \mu \mathrm{g} \mathrm{mL}^{-1}$ kanamycin per well. After two days of growth, an equal volume of $40 \% \mathrm{w} / \mathrm{v}$ glucose was added to saturated cultures for a final solution of $20 \% \mathrm{w} / \mathrm{v}$ glucose. All dispensing into microplates was done with a 96-channel pipette. The amount of glucose needed to lower the $\mathrm{pH}$ below 2.3 via the production of gluconic acid was estimated to be $13 \% \mathrm{w} / \mathrm{v}$, but the higher concentration was used to account for any use of glucose as a carbon source and still maintain an excess amount. Viability tests demonstrated that the bacteria were still viable after two days of culture in such a solution.

Bacteria were incubated with glucose for $48 \mathrm{~h}$ to allow acid production to reach completion. Plates were then centrifuged for $3 \mathrm{~min}$ at $3200 \times \mathrm{g}$ (top speed), and $90 \mu \mathrm{L}$ of the biolixiviant supernatant was removed and added to $10 \mu \mathrm{L}$ TB at a final concentration of $40 \mu \mathrm{g} \mathrm{mL}^{-1}$. After $1 \mathrm{~min}$ of mixing by vortexing, absorbance was measured for each well at 435 and $545 \mathrm{~nm}$ on a Synergy 2 plate reader (Biotek Instruments, Winooski, VT, USA). Because of the variation in background absorbance from well to well on each plate, absorbance was measured at these two wavelengths, and their ratio was used as a proxy for $\mathrm{pH}$, which correlates linearly within the range of $\mathrm{pH}$ for the majority of biolixiviants produced by the collection (Supplementary Fig. 1).

Bromophenol blue acidification rate screen. Acidification rate was measured using the $\mathrm{pH}$-indicating dye bromophenol blue (BPB; 161-0404, Bio-Rad, Richmond, CA). Knockout collection strains were grown for two days. OD was measured at $590 \mathrm{~nm}$ for each well, and then $5 \mu \mathrm{L}$ of culture was transferred to a polystyrene assay plate containing $95 \mu \mathrm{L}$ of $2 \% \mathrm{w} / \mathrm{v}$ glucose and $20 \mu \mathrm{g} \mathrm{mL}-1$ BPB in deionized water per well. The initial $\mathrm{pH}$ of the culture is just above 5 , and within moments of adding culture to glucose with $\mathrm{BPB}$, the color begins to change rapidly. Assay plates were mixed by vortexing for one minute after the addition of bacterial culture and then immediately transferred to a plate reader, where the change in color was tracked by measuring absorbance at $600 \mathrm{~nm}$ every minute for 6 min, resulting in seven reads. Mean rate $(V)$ and R-squared were calculated by the Gen5 microplate reader and imager software (Biotek Instruments). A plot of all $V$ relative to OD demonstrated that the two are correlated; thus, $V$ was normalized to OD for each well (Supplementary Fig. 3).

Hit identification in acidification end point and rate screens. Once every well had its assigned data point (A435/A545 for TB and V/OD for BPB), hits were determined by first identifying outliers for each plate. The interquartile range and upper and lower bounds were calculated in Microsoft Excel considering all wells with cultured disruption strains. Any data point that was more than $1.5 \times$ the interquartile range below or above the first and third quartile, respectively, was considered an outlier. A disruption strain was considered a hit if over half of the wells for that strain (or 1 of 2) were outliers.

End point acidity and rate quantification with colorimetric dyes. For each assay, knockout strains identified as hits were isolated from the knockout collection into new microplates, along with several blanks per plate, and proxy wild-type strainsGoB58 strains with an intergenic transposon insertion that should not affect the acidification phenotype (see next "Methods" section). Replicates for each knockout strain were distributed across several plates to control for any slight differences between assay plates. For purposes of comparison, all proxy WT strains were considered together; however, OD and acidification phenotypes were measured for each proxy WT strain separately to verify that growth and acidification were unaffected in these strains (Supplementary Fig. 4).

Acidification phenotypes for the disruption strains (for $n$, see Supplementary Data 6C and D) were compared to that of proxy WT (TB, $n=144$; BPB, $n=31$ ) with Student's $t$-test in Microsoft Excel, two-tailed assuming equal variances. For the acidification rate assay, V/OD data was discarded if the $R^{2}$ for $V$ was below 0.5 . A Bonferroni correction was used to determine significance to account for the possibility a comparison is significant by chance alone: a phenotype was considered significant if $p<0.05 / N$, where $N$ is the number of comparisons being made $(N=120$ or $N=242$ for endpoint acidity comparisons with pWT set A or set B, respectively; $N=60$ for rate of acidification comparisons with $\mathrm{pWT}$ ).

\section{Choice of proxy wild-type comparison. The biolixiviant end point $\mathrm{pH}$ and} acidification rate of each G. oxydans mutant were compared against a proxy wildtype set of mutants for each phenotype. To account for the presence of a kanamycin cassette in the genome, the proxy wild-type set for each phenotype was constructed of several mutants with the transposon inserted in an intergenic region that had no growth defect and no apparent change in phenotype (Supplementary Fig. 2).

As the efficiency of E. coli WM3064 to G. oxydans mating was low, construction of the G. oxydans progenitor collection required 18 mating batches. As a result, there were slight variations in the wild-type background from batch to batch, notably two distinct saturated culture densities throughout the collection.
For the acidification rate, these variations did not affect the wild-type behavior across the collection due to normalization with culture density, and a single set of proxy wild-type strains could be used as a comparison with disruption strains in the quantification assays. For the end point $\mathrm{pH}$ measurement with $\mathrm{TB}$, these two distinct growth phenotypes were found to affect the result, and thus a proxy wildtype set was used for each. Density measurements across the knockout collection revealed sets of plates resembling each phenotype. Proxy wild-type set A was used as a comparison for insertion mutants located in plates 1-76, 110-130, and $160-185$ of the condensed collection (Supplementary Fig. 2A), and proxy wild-type set $\mathrm{B}$, which had a lesser saturated culture density, was used for mutants in plates 77-109 and 130-159 (Supplementary Fig. 2B).

Optical density after 2 days of growth and endpoint acidity using the TB absorbance ratio (A435/A545) were compared for both wild-type sets (Supplementary Fig. 2). For wild-type set A, which was used for the BPB quantification assay, acidification rate of individual proxy WT strains was also compared. Pairwise comparisons were all made using the emmeans package in $\mathrm{R}$ with a Tukey $p$-value adjustment (https://github.com/rvlenth/emmeans).

Direct measurement of biolixiviant pH. Bacteria were grown for $48 \mathrm{~h}$ in culture tubes containing $4 \mathrm{~mL}$ YPM with $100 \mu \mathrm{g} \mathrm{mL} \mathrm{m}^{-1}$ kanamycin. One tube was left uninoculated as a no-bacteria control. OD was normalized to 1.9 and diluted in half with $40 \%$ glucose for a final $20 \%$ solution in $1.5 \mathrm{~mL}$. Five replicates were created for each strain and control, and all mixtures were randomly distributed across two 96-well deep well plates. Seven hundred and fifty microliter of mixture was transferred from each well to a second set of deep-well plates for bioleaching experiments. All plates were incubated with shaking at $800 \mathrm{rpm}$ at room temperature.

After two days, one set of deep-well plates was centrifuged for $10 \mathrm{~min}$ at $3200 \times \mathrm{g}$ (top speed), and the $\mathrm{pH}$ of the supernatant was measured by insertion of a microprobe to the same depth in each well.

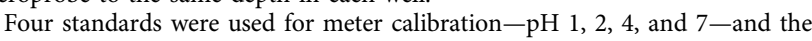
meter was recalibrated after every 12 measurements. $\mathrm{pH}$ measurements for each disruption strain $(n=5)$ were compared with those of proxy WT $(n=15)$ using Student's $t$-test in Microsoft Excel, two-tailed, with equal variance. A biolixiviant $\mathrm{pH}$ was considered significantly different if $p<0.05 / N$, with $N=22$.

Direct measurement of REE bioleaching. The second set of deep-well plates was centrifuged for $10 \mathrm{~min}$ at $3200 \times \mathrm{g}$ (top speed), and $500 \mu \mathrm{L}$ of biolixiviant was transferred from each well to a $1.7 \mathrm{~mL}$ Eppendorf tube. Twenty milligrams $(4 \% \mathrm{w} /$ v) of retorted phosphor powder (gift from Idaho National $\mathrm{Lab}^{26}$ ) was added to each tube for bioleaching. Tubes were shaken horizontally for $36 \mathrm{~h}$ at room temperature and then centrifuged to pellet the remaining solids. Supernatant with leached REE was filtered through a $0.45 \mu \mathrm{m}$ AcroPrep Advance 96-well filter plate (8029, Pall Corporation, Show Low, AZ, USA) by centrifugation at $1500 \times g$ for $5 \mathrm{~min}$.

All samples were diluted 1/200 in 2\% trace metal grade nitric acid (JT9368, J.T. Baker, Radnor, PA) and analyzed by an Agilent 7800 ICP-MS for all REE concentrations (m/z: Sc, 45; Y, 89; La, 139; Ce, 140; Pr, 141; Nd, 146; Sm, 147; Eu, 153; Gd, 157; Tb, 159; Dy, 163; Ho, 165; Er, 166; Tm, 169; Yb, 172; and Lu, 175) using a rare earth element mix standard (67349, Sigma-Aldrich, St. Louis, MO) and a rhodium in-line internal standard (SKU04736, Sigma-Aldrich, St. Louis, MO, $m$ / $z=103$ ). Quality control was performed by periodic measurement of standards, blanks, and repeat samples. A pWT biolixiviant sample without bioleaching was spiked with $100 \mathrm{ppb}$ REE standard and analyzed for all REE concentrations as a control. ICP-MS data were analyzed using the program MassHunter, version 4.5.

An additional $1 / 20$ dilution in $2 \%$ nitric acid was analyzed for $\delta m g d h$ and $\delta p q q c$ disruption strains and the no-bacteria control (glucose).

Bioleaching measurements for each disruption strain $(n=5)$ were compared with those of proxy WT $(n=15)$ or glucose $(n=5)$ using Student's $t$-test in Microsoft Excel, two-tailed, assuming equal variances. Total REE extracted was considered significantly different if $p<0.05 / N$, with $N=12$ for those compared to pWT and $N=2$ for those compared to glucose.

Reporting summary. Further information on research design is available in the Nature Research Reporting Summary linked to this article.

\section{Data availability}

Experimental data generated in this study are provided in the Supplementary Information/Source Data file. Raw sequencing data are available through Cornell University eCommons: https://doi.org/10.7298/7s81-5t81. The assembled G. oxydans NRRL B58 genome has been deposited at DDBJ/ENA/GenBank under the accession JAIPVW000000000. The version described in this paper is version JAIPVW010000000

\section{Material availability}

Correspondence and material requests should be addressed to B.B. Individual strains (up to $\approx 10$ at a time) are available at no charge for academic researchers. We are happy to supply a duplicate of the entire G. oxydans knockout collection to academic researchers but will require reimbursement for materials, supplies and labor costs. Commercial researchers should contact Cornell Technology Licensing for licensing details. 


\section{Code availability}

The general purpose Knockout Sudoku software is available at https://doi.org/10.5281/ zenodo.5546671. A specific version of the Knockout Sudoku software and input files for creation of the G. oxydans knockout collection is available at https://doi.org/10.5281/ zenodo.5585726.

Received: 22 July 2021; Accepted: 27 October 2021; Published online: 18 November 2021

\section{References}

1. Bauer, D. et al. Critical Materials Strategy (U.S. Department of Energy, 2011).

2. Grasso, V. B. Rare earth elements in national defense: background, oversight issues, and options for congress. Report No. R41744, (Congressional Research Service, 2013).

3. Humphries, M. Rare earth elements: the global supply chain. Report No. R41347, (Congressional Research Service, 2013).

4. Dent, P. C. Rare earth elements and permanent magnets. J. Appl. Phys. 111, 07A721 (2012).

5. Nazarov, M. \& Noh, D. New Generation of Europium-Activated and TerbiumActivated Phosphors (Pan Stanford Publishing, 2011).

6. Müller, T. \& Friedrich, B. Development of a recycling process for nickel-metal hydride batteries. J. Power Sources 158, 1498-1509 (2006).

7. Schubert, E. F. \& Kim, J. K. Solid-state light sources getting smart. Science $\mathbf{3 0 8}$ 1274-1278 (2005).

8. Norman, A. F., Prangnell, P. B. \& McEwen, R. S. The solidification behaviour of dilute aluminium-scandium alloys. Acta Mater. 46, 5715-5732 (1998).

9. Adesina, O., Anzai, I. A., Avalos, J. L. \& Barstow, B. Embracing biological solutions to the sustainable energy challenge. Chem 2, 20-51 (2017).

10. Global Rare Earth Materials Market, Forecast to 2025. (Frost \& Sullivan, 2019).

11. Annual Energy Outlook 2020, with Projections to 2050. (U.S. Energy Information Administration, Office of Energy Analysis, U.S. Department of Energy, 2020).

12. Voncken, J. H. L. The rare earth elements: an introduction. Springerbriefs Earth Sci. https://doi.org/10.1007/978-3-319-26809-5 (2016).

13. Scheyder, E. China Set to Control Rare Earth Supply for Years Due to Processing Dominance, https://www.reuters.com/article/us-china-usarareearth-refining/china-set-to-control-rare-earth-supply-for-years-due-toprocessing-dominance-idUSKCN1T004J (2019).

14. Mining the Future: How China is Set to Dominate the Next Industrial Revolution (Foreign Policy, 2019).

15. Bonificio, W. D. \& Clarke, D. R. Rare-earth separation using bacteria. Environ. Sci. Technol. Lett. 3, 180-184 (2016).

16. Brisson, V. L., Zhuang, W.-Q. \& Alvarez-Cohen, L. Bioleaching of rare earth elements from monazite sand. Biotechnol. Bioeng. 113, 339-348 (2016)

17. Park, D. M. et al. Bioadsorption of rare earth elements through cell surface display of lanthanide binding tags. Environ. Sci. Technol. 50, 2735-2742 (2016).

18. Jin, H. et al. Techno-economic assessment for integrating biosorption into rare earth recovery process. ACS Sustain. Chem. Eng. 5, 10148-10155 (2017).

19. Thompson, V.S. et al. Techno-economic and life cycle analysis for bioleaching rare-earth elements from waste materials. ACS Sustain. Chem. Eng. 6, 1602-1609 (2018).

20. Johnson, D. B. Biomining-biotechnologies for extracting and recovering metals from ores and waste materials. Curr. Opin. Biotechnol. 30, 24-31 (2014).

21. Brierley, C. L. Innovative process development in metallurgical industry. Concept Comm. 6, 109-135 (2016).

22. Valdés, J. et al. Acidithiobacillus ferrooxidans metabolism: from genome sequence to industrial applications. BMC Genom. 9, 597 (2008).

23. Gentina, J. C. \& Acevedo, F. Copper bioleaching in Chile. Minerals 6, 23 (2016).

24. Merritt, R. R. High temperature methods for processing monazite: I. Reaction with calcium chloride and calcium carbonate. J. Less Common Met. 166, 197-210 (1990).

25. Peelman, S., Sun, Z. H. I., Sietsma, J. \& Yang, Y. In Leaching of Rare Earth Elements: Review of Past and Present Technologies. (eds. Lima, I. B. D., Filho, W. L.) Rare Earths Industry, 319-334 https://doi.org/10.1016/B978-0-12802328-0.00021-8 (Elsevier, 2016).

26. Reed, D. W., Fujita, Y., Daubaras, D. L., Jiao, Y. \& Thompson, V. S. Bioleaching of rare earth elements from waste phosphors and cracking catalysts. Hydrometallurgy 166, 34-40 (2016).

27. Rasoulnia, P., Barthen, R. \& Lakaniemi, A.-M. A critical review of bioleaching of rare earth elements: the mechanisms and effect of process parameters. Crit. Rev. Environ. Sci. Technol. 51, 1-50 (2020).

28. Capeness, M. J. \& Horsfall, L. E. Synthetic biology approaches towards the recycling of metals from the environment. Biochem. Soc. Trans. 48, 1367-1378 (2020).
29. Schleyer, U., Bringer-Meyer, S. \& Sahm, H. An easy cloning and expression vector system for Gluconobacter oxydans. Int. J. Food Microbiol. 125, 91-95 (2008).

30. Kallnik, V., Meyer, M., Deppenmeier, U. \& Schweiger, P. Construction of expression vectors for protein production in Gluconobacter oxydans. J. Biotechnol. 150, 460-465 (2010).

31. Kostner, D., Peters, B., Mientus, M., Liebl, W. \& Ehrenreich, A. Importance of codB for new codA-based markerless gene deletion in Gluconobacter strains. Appl. Microbiol. Biotechnol. 97, 8341-8349 (2013).

32. Fricke, P. M. et al. A tunable l-arabinose-inducible expression plasmid for the acetic acid bacterium Gluconobacter oxydans. Appl. Microbiol. Biotechnol. 104, 9267-9282 (2020).

33. Qin, Z. et al. Repurposing the endogenous type I-E CRISPR/Cas system for gene repression in Gluconobacter oxydans WSH-003. ACS Synth. Biol. 10, 84-93 (2021).

34. Chen, Y. et al. Identification of gradient promoters of Gluconobacter oxydans and their applications in the biosynthesis of 2-keto-L-gulonic acid. Front. Bioeng. Biotechnol. 9, 673844 (2021).

35. Gupta, A., Verma, V. \& Qazi, G. N. Transposon induced mutation in Gluconobacter oxydans with special reference to its direct-glucose oxidation metabolism. FEMS Microbiol. Lett. 147, 181-188 (1997).

36. Baym, M., Shaket, L., Anzai, I. A., Adesina, O. \& Barstow, B. Rapid construction of a whole-genome transposon insertion collection for Shewanella oneidensis by Knockout Sudoku. Nat. Commun. 7, 13270 (2016).

37. Anzai, I. A., Shaket, L., Adesina, O., Baym, M. \& Barstow, B. Rapid curation of gene disruption collections using Knockout Sudoku. Nat. Protoc. 12, 2110-2137 (2017).

38. Krajewski, V. et al. Metabolic engineering of gluconobacter oxydans for improved growth rate and growth yield on glucose by elimination of gluconate formation $\nabla$. Appl. Environ. Microbiol. 76, 4369-4376 (2010).

39. Shin, D., Kim, J., Kim, B.-s, Jeong, J. \& Lee, J.-c. Use of Phosphate solubilizing bacteria to leach rare earth elements from monazite-bearing ore. Minerals 5, 189-202 (2015).

40. Rodríguez, H. \& Fraga, R. Phosphate solubilizing bacteria and their role in plant growth promotion. Biotechnol. Adv. 17, 319-339 (1999).

41. Hsieh, Y.-J. \& Wanner, B. L. Global regulation by the seven-component Pi signaling system. Curr. Opin. Microbiol. 13, 198-203 (2010).

42. Hölscher, T. \& Görisch, H. Knockout and overexpression of pyrroloquinoline quinone biosynthetic genes in Gluconobacter oxydans $621 \mathrm{H} \nabla$. J. Bacteriol. 188, 7668-7676 (2006).

43. Ghilarov, D. et al. The origins of specificity in the microcin-processing protease TldD/E. Structure 25, 1549-1561 (2017).

44. Martins, A. M. et al. A two-component protease in Methylorubrum extorquens with high activity toward the peptide precursor of the redox cofactor pyrroloquinoline quinone. J. Biol. Chem. 294, 15025-15036 (2019).

45. Muynck, C. D. et al. The genus Gluconobacter oxydans: comprehensive overview of biochemistry and biotechnological applications. Crit. Rev. Biotechnol. 27, 147-171 (2008).

46. Richhardt, J., Bringer, S. \& Bott, M. Mutational analysis of the pentose phosphate and Entner-Doudoroff pathways in Gluconobacter oxydans reveals improved growth of a $\Delta$ edd $\Delta$ eda mutant on mannitol. Appl. Environ. Microbiol. 78, 6975-6986 (2012).

47. Matsutani, M. \& Yakushi, T. Pyrroloquinoline quinone-dependent dehydrogenases of acetic acid bacteria. Appl. Microbiol. Biotechnol. 102, 9531-9540 (2018).

48. Liu, D., Ke, X., Hu, Z.-C. \& Zheng, Y.-G. Improvement of pyrroloquinoline quinone-dependent d-sorbitol dehydrogenase activity from Gluconobacter oxydans via expression of Vitreoscilla hemoglobin and regulation of dissolved oxygen tension for the biosynthesis of 6-(N-hydroxyethyl)-amino-6-deoxy-a1-sorbofuranose. J. Biosci. Bioeng. 131, 518-524 (2021).

49. Liu, D., Ke, X., Hu, Z.-C. \& Zheng, Y.-G. Combinational expression of $\mathrm{D}$-sorbitol dehydrogenase and pyrroloquinoline quinone increases 6- $(\mathrm{N}$ hydroxyethyl)-amino-6-deoxy-a-L-sorbofuranose production by Gluconobacter oxydans through cofactor manipulation. Enzym. Microb. Technol. 141, 109670 (2020).

50. Wang, P. et al. Overexpression of pyrroloquinoline quinone biosynthetic genes affects l-sorbose production in Gluconobacter oxydans WSH-003. Biochem. Eng. J. 112, 70-77 (2016).

51. Yuan, J., Wu, M., Lin, J. \& Yang, L. Combinatorial metabolic engineering of industrial Gluconobacter oxydans DSM2343 for boosting 5-keto-D-gluconic acid accumulation. BMC Biotechnol. 16, 42 (2016).

52. Misra, H. S., Rajpurohit, Y. S. \& Khairnar, N. P. Pyrroloquinoline-quinone and its versatile roles in biological processes. J. Biosci. 37, 313-325 (2012).

53. Bolger, A. M., Lohse, M. \& Usadel, B. Trimmomatic: a flexible trimmer for Illumina sequence data. Bioinformatics 30, 2114-2120 (2014).

54. Bankevich, A. et al. SPAdes: a new genome assembly algorithm and its applications to single-cell sequencing. J. Comput. Biol. 19, 455-477 (2012).

55. Gurevich, A., Saveliev, V., Vyahhi, N. \& Tesler, G. QUAST: quality assessment tool for genome assemblies. Bioinformatics 29, 1072-1075 (2013). 
56. Seppey, M., Manni, M. \& Zdobnov, E. M. Gene prediction, methods and protocols. Methods Mol. Biol. 1962, 227-245 (2019).

57. Brettin, T. et al. RASTtk: a modular and extensible implementation of the RAST algorithm for building custom annotation pipelines and annotating batches of genomes. Sci. Rep. 5, 8365 (2015).

58. Overbeek, R. et al. The SEED and the Rapid Annotation of microbial genomes using Subsystems Technology (RAST). Nucleic Acids Res. 42, D206-D214 (2014).

59. Aziz, R. K. et al. The RAST Server: rapid annotations using subsystems technology. BMC Genomics 9, 75 (2008).

60. Buchfink, B., Reuter, K. \& Drost, H.-G. Sensitive protein alignments at tree-oflife scale using DIAMOND. Nat. Methods 18, 366-368 (2021).

61. Jones, P. et al. InterProScan 5: genome-scale protein function classification. Bioinformatics 30, 1236-1240 (2014).

62. Götz, S. et al. High-throughput functional annotation and data mining with the Blast2GO suite. Nucleic Acids Res. 36, 3420-3435 (2008).

63. Adrian, A. \& Jörg, R. Gene Set Enrichment Analysis with topGO. http:// compdiag.molgen.mpg.de/ngfn/docs/2007/sep/topGO_Exercises.pdf (2007).

\section{Acknowledgements}

We thank D. Reed and Y. Fujita at Idaho National Lab for advice and for the gift of retorted phosphor powder. A.M.S. was supported by a Cornell Energy Systems Institute Postdoctoral Fellowship and a Small Grant from the Cornell Atkinson Center for Sustainability. This work was supported by Cornell University startup funds, an Academic Venture Fund award from the Atkinson Center for Sustainability at Cornell University, a Career Award at the Scientific Interface from the Burroughs Welcome Fund to B.B. and by ARPA-E award DE-AR0001341 to B.B., E.G. and M.W.

\section{Author contributions}

Conceptualization, A.M.S. and B.B.; Methodology, A.M.S. and B.B.; Investigation, A.M.S., B.P., S.M. and B.B; Writing-Original draft, A.M.S. and B.B.; Writing-Review and editing, A.M.S., B.P., S.M., M.R., M.W., E.G. and B.B.; Funding acquisition, A.M.S., E.G., M.W. and B.B.; Resources, M.R., E.G. and B.B.; Supervision, M.R. and B.B.; Data curation, A.M.S. and B.B.; Visualization, A.M.S. and B.B.; Formal analysis, A.M.S. and S.M.

\section{Competing interests}

The authors A.M.S, B.P., S.M. and B.B. are pursuing patent protection for engineered organisms using knowledge gathered in this work (US provisional application 63/ $220,475)$. The remaining authors declare no competing interests.

\section{Additional information}

Supplementary information The online version contains supplementary material available at https://doi.org/10.1038/s41467-021-27047-4.

Correspondence and requests for materials should be addressed to Buz Barstow.

Peer review information Nature Communications thanks the anonymous reviewers for their contribution to the peer review of this work. Peer reviewer reports are available.

Reprints and permission information is available at http://www.nature.com/reprints

Publisher's note Springer Nature remains neutral with regard to jurisdictional claims in published maps and institutional affiliations.

cc (i) Open Access This article is licensed under a Creative Commons Attribution 4.0 International License, which permits use, sharing, adaptation, distribution and reproduction in any medium or format, as long as you give appropriate credit to the original author(s) and the source, provide a link to the Creative Commons license, and indicate if changes were made. The images or other third party material in this article are included in the article's Creative Commons license, unless indicated otherwise in a credit line to the material. If material is not included in the article's Creative Commons license and your intended use is not permitted by statutory regulation or exceeds the permitted use, you will need to obtain permission directly from the copyright holder. To view a copy of this license, visit http://creativecommons.org/ licenses/by/4.0/.

(C) The Author(s) 2021 